\title{
An XFEM-based numerical scheme to compute crack-induced electrical resistivity changes in cracked CNT-reinforced composites using ANSYS
}

\author{
José Vargas, ${ }^{1, \text { a) }}$ Luis Rodríguez-Tembleque, ${ }^{1, b)}$ Federico C. Buroni, ${ }^{1,}$ c) Enrique \\ García-Macías, ${ }^{2, \text { d) }}$ and Andrés Sáez ${ }^{1, \text { e) }}$ \\ 1) Escuela Técnica Superior de Ingeniería, Universidad de Sevilla, \\ Camino de los Descubrimientos s/n, Sevilla 41092, Spain. \\ ${ }^{2)}$ Escuela Técnica Superior de Ingeniería de Caminos, Canales y Puertos \\ Campus Universitario de Fuentenueva, Granada 18071, Spain. \\ a)Electronic mail: josvarcru@gmail.com \\ b)Corresponding author: luisroteso@us.es \\ c)Electronic mail: fburoni@us.es \\ d)Electronic mail: enriquegm@ugr.es \\ e)Electronic mail: andres@us.es
}

\begin{abstract}
This paper presents an eXtended Finite Element Method (XFEM)-based numerical scheme to compute electrical resistivity changes caused by the presence of cracks. Using the commercial finite element package ANSYS, the virtual continuous monitoring of the structure is solved in two steps. First, the strain response of the cracked composite domain is computed by means of the XFEM. In the second step, the electrical conductivity of the piezorresistive elements located in the domain are updated according to the strain state and the electric resistance between two electrodes of the damaged plate is computed. The comparison with the electric resistance measured for the undamaged plate allows us to detect the presence of a crack and its severity. Several numerical studies are provided to show the capabilities of this computational framework.
\end{abstract}

\section{INTRODUCTION}

In recent years, self-sensing structural materials have focused the attention of the scientific community due to their potential applications in the continuous monitoring of the integrity of structures. In this context, Carbon nanotube (CNT)-reinforced composites have exhibited a piezoresistive behavior that permits their use as sensors in novel structural health monitoring (SHM) applications [1, 2, 3, 4, 5, 6, 7, 8] by measuring the electrical resistivity change of the CNT-reinforced composite structure.

This paper presents an eXtended Finite Element Method (XFEM)-based numerical scheme to compute electrical resistivity changes caused by the presence of cracks in a damage domain. The methodology, which was previously presented in [9], is noe developed in a more compact and robust finite element computational framework. Using the commercial finite element package ANSYS, the virtual continuous monitoring of the structure is solved in two steps. First, the the strain response of the cracked composite domain are computed by means of the XFEM. In the second step, the electrical conductivity of the piezorresistive elements located in the domain are updated according to the strain state and the electric resistance between two electrodes of the damaged plate is computed. The comparison with the electric resistance measured for the undamaged plate allows us to detect the presence of a crack and its severity. To show the capabilities of this virtual health monitoring scheme, several numerical studies are presented.

\section{VIRTUAL CRACK DETECTION SETTING}

For this purpose, a MWCNT/epoxy composite square plate $(2 L \times 2 L \times t, L=100 \mathrm{~mm}$ and $t=2 \mathrm{~mm})$ plate under uniform stress $\left(\bar{\sigma}_{y y}=1 \mathrm{MPa}\right)$ is studied (see Fig.1). The plate presents a finite straight inclined crack whose length is $2 a$. In this example, we have considered a MWCNT/epoxy composite with filler content moderately far from the percolation threshold ( $f_{c} \approx 0.3 \mathrm{wt} \%$ ), namely $f_{c}=0.5 \mathrm{wt} \%$. In this manner, we ensure: (i) a linear strain sensitivity of the plate, and (ii) the accuracy of the approach implemented to model the piezoresistive behavior of the composite $[8,10]$. Based on [8], the values of the material constants are given in Table I.

Fracture and Damage Mechanics

AIP Conf. Proc. 2309, 020004-1-020004-6; https://doi.org/10.1063/5.0033981

Published by AIP Publishing. 978-0-7354-4045-6/\$30.00 


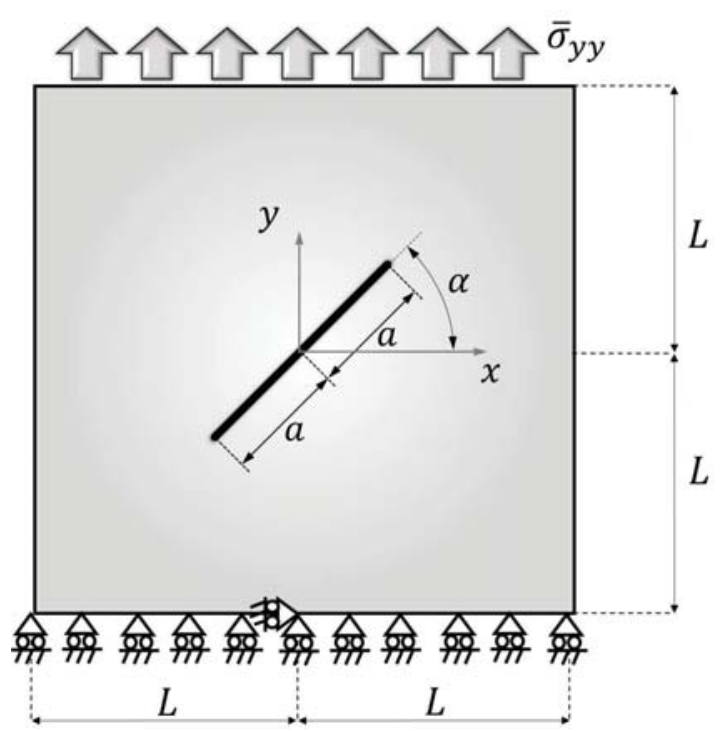

(a)

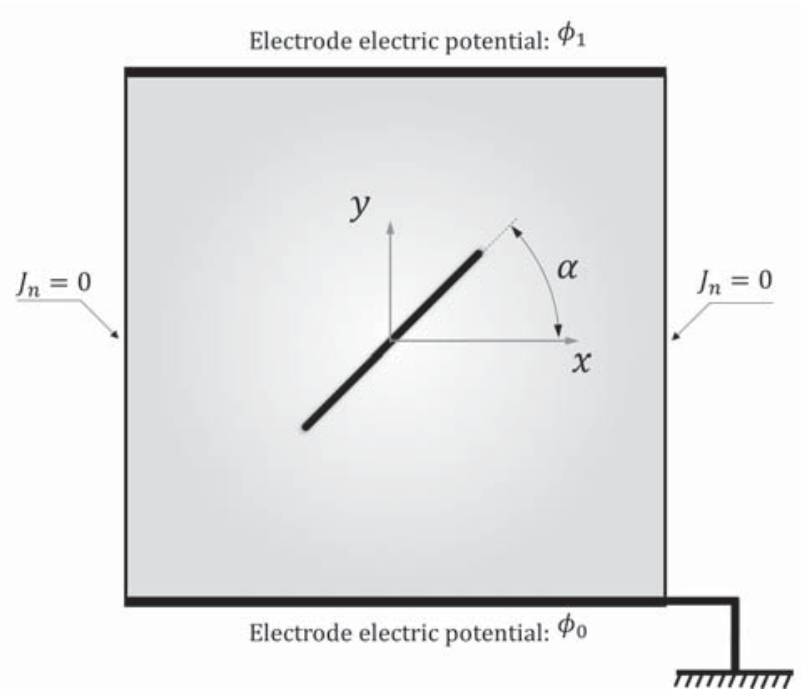

(b)

FIGURE 1. (a) Cracked plate under uniform stress $\left(\bar{\sigma}_{y y}\right)$. (b) Electrodes position in the cracked MWCNT/epoxy composite plate.

TABLE I. Effective electromechanical properties of smart MWCNT/epoxy strip-like sensor [7, 8].

\begin{tabular}{lcccccc}
\hline \hline $\mathrm{wt} \%$ & $E(G P a)$ & $v$ & $\kappa\left(S m^{-1}\right)$ & $\lambda_{11}$ & $\lambda_{12}$ & $\lambda_{44}$ \\
\hline 0.5 & 2.86 & 0.28 & $1.22 \times 10^{-2}$ & 6.84 & 7.99 & 1.19 \\
\hline \hline
\end{tabular}

The XFEM has been used to solve the mechanical problem, i.e., the strain response of the cracked plate. In the XFEM [11, 12, 13], the crack discontinuity is modelled independent to the finite element mesh using a set of specific local enrichment functions derived from the theoretical context of fracture mechanics. The use of these special elements allow us to handle the crack tip asymptotic stresses. Using the commercial finite element package ANSYS [14], the analysis have been developed using the Singularity-Based Method, so the displacement functions in the FEM formulation are enhanced by introducing additional enrichment functions that capture the jump in displacement across the crack surface and also the crack-tip singularities. So, once the strain field is known in the cracked domain, the relative resistivity changes $\left(\mathbf{r}=\Delta \rho / \rho_{o}\right)$ can be computed as: $\mathbf{r}=\Pi \varepsilon$, i.e.,

$$
\left[\begin{array}{c}
\Delta \rho_{1} / \rho_{o} \\
\Delta \rho_{2} / \rho_{o} \\
\Delta \rho_{6} / \rho_{o}
\end{array}\right]=\left[\begin{array}{ccc}
\lambda_{11} & \lambda_{12} & 0 \\
\lambda_{12} & \lambda_{22} & 0 \\
0 & 0 & \lambda_{44}
\end{array}\right]\left[\begin{array}{c}
\varepsilon_{11} \\
\varepsilon_{22} \\
2 \varepsilon_{12}
\end{array}\right]
$$

and electric conductivity tensor $\kappa$ can be obtained as $\kappa=\rho^{-1}$, being $\rho$ the electric resistivity computed according to: $\boldsymbol{\rho}=\rho_{o}(\mathbf{I}+\mathbf{r})=\rho_{o}(\mathbf{I}+\Pi \varepsilon)$. In Eq.(1), the terms $\lambda_{i j}$ denote the piezoresistivity coefficients. Specifically, $\lambda_{11}$ represents the longitudinal piezoresistive effect, $\lambda_{12}$ relates the transverse piezoresistive effect, and $\lambda_{44}$ describes the effect on an out-of-plane electric field by the change of the in-plane current induced by in-plane shear stress. All the piezoresistivity coefficients $\lambda_{i j}$ can be obtained by two virtual experiments using the previously outlined micromechanics approach, including a laterally constrained uni-axial dilation test and a distortion test (interested readers may refer to [10] for further theoretical details).

Afterward, the electric potential distribution is obtained by solving the electrostatic problem in absence of spacecharge density: $\nabla \cdot(\kappa \nabla \phi)=0$, using ANSYS. Adopting the electrodes configuration presented in Fig.1, one simple way to quantify the electric resistivity changes induced by the presence of a crack in the plate would be to evaluate the electric resistance between electrodes $(R)$ of the damaged plate and compare its value with the electric resistance measured for the undamaged plate $\left(R_{O}\right)$. The electric resistance $(R)$ of a plate of thickness $t$ can be computed as

$$
R=\frac{\Delta \phi}{I}
$$




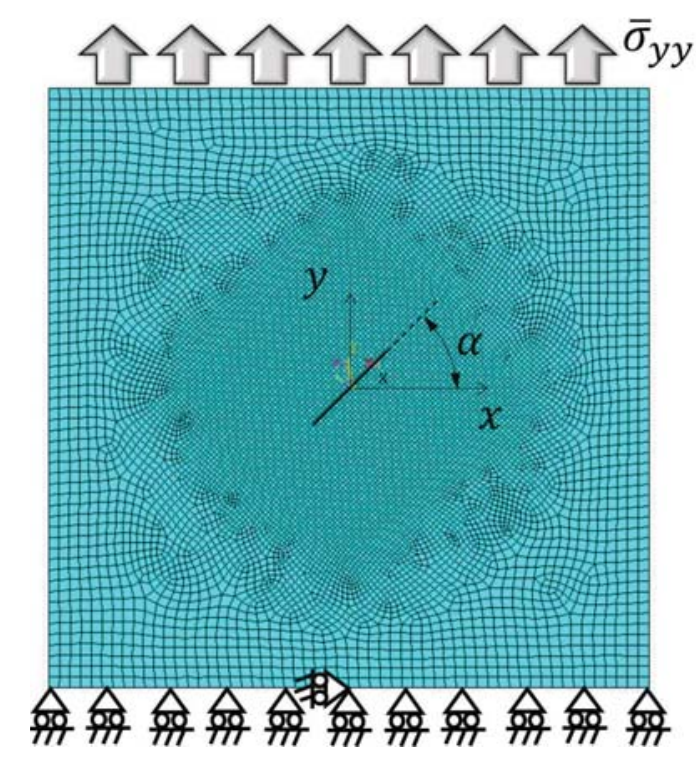

FIGURE 2. Mesh details for the stationary crack analysis using the XFEM.

where $\Delta \phi=\phi_{1}-\phi_{0}$ is the voltage difference recorded from electrodes and $I$ is the electric current flowing through the plate, which may be computed from the electric flux density $\mathbf{J}(\mathbf{J}=-\boldsymbol{\kappa} \nabla \phi)$ as

$$
I=\int_{A} \mathbf{J} \cdot \mathbf{n} d A \approx \int_{-l}^{+l} \mathbf{J} \cdot \mathbf{n} t d x
$$

being $\mathbf{n}$ the outward facing surface normal vector.

\section{CRACK DETECTION: NUMERICAL STUDIES}

The proposed virtual health monitoring scheme presented in this work (see Fig. 1) is applied to detect damage in a cracked plate due to several crack configurations. Firstly, a stationary crack analysis using the XFEM is solved to show the accuracy of the finite element mesh adopted (see Fig. 2), where $L / a=5$. In Fig. 3, the influence of the crack orientation $(\alpha)$ on the stress intensity factors: $K_{I} / K_{o}$ and $K_{I I} / K_{o}$ (being $K_{o}=\bar{\sigma}_{y y} \sqrt{\pi a}$ ) is presented by comparison with the theoretical solution [15]. These results show an excellent agreement.

\section{Resistance changes: influence of the crack size}

After the validation, we are going to study the influence of the crack size on the electric resistance changes in the damaged plate. So, in this example, the influence of the crack size parameter $(L / a)$ on the electric resistance is presented in Fig. 4, taking into account the several orientations of the crack. Results are computed under crack-face electric impermeable conditions. We can see how the resistance $(R)$ increases with the crack size $(a)$, or in other words, with the decrease of the ratio $L / a$ (see Fig.4). Moreover, the resistance $(R)$ also increases with angle $\alpha$ (the orientation of the crack relative to the electrodes). Fig. 5 shows the electric potential distributions for $L / a=2.5$ and the crack orientations: $\alpha=0^{\circ}$ and $\alpha=45^{\circ}$. So, it is clear that the electric field is affected by the crack size and the crack orientation. 


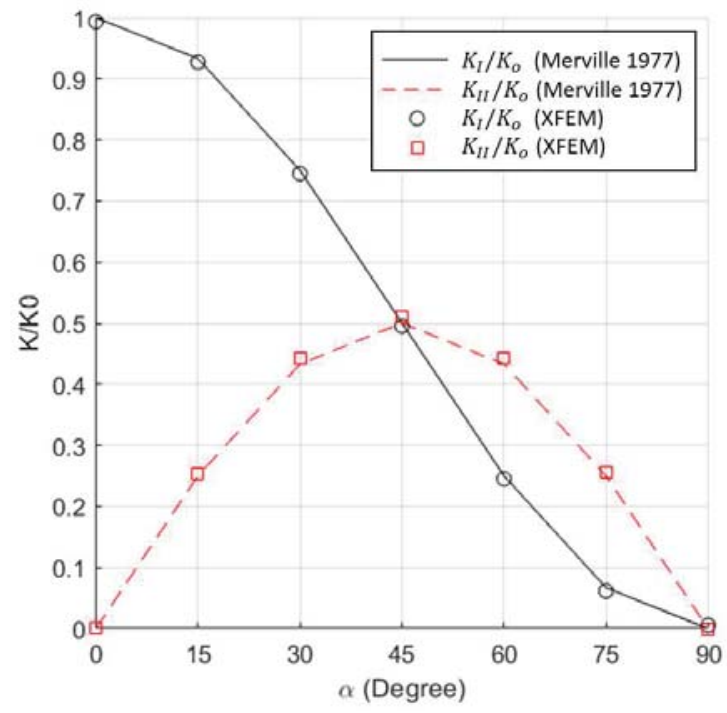

FIGURE 3. Influence of the crack orientation $(\alpha)$ on the stress intensity factors: $K_{I} / K_{o}$ and $K_{I I} / K_{o}$, being $K_{o}=\bar{\sigma}_{y y} \sqrt{\pi a}$.

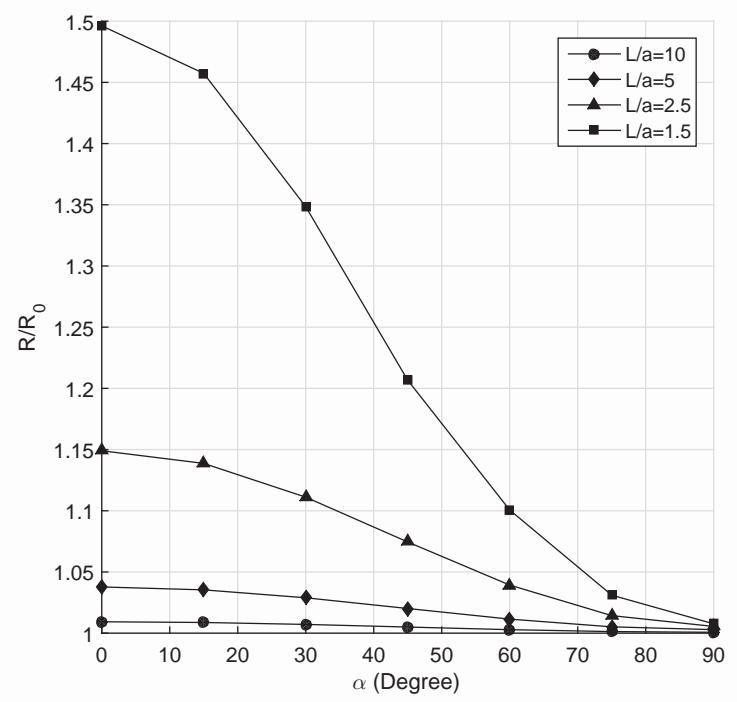

FIGURE 4. Influence of the size of the crack $(L / a)$ and the crack orientation $(\alpha)$ on the electric resistance changes.

\section{Resistance changes: influence of the electric permittivity crack conditions}

To conclude this section, it is important to consider the crack-face electric permittivity conditions, i.e., permeable, impermeable or semipermeable crack conditions. The crack permittivity is designed as $\kappa_{c}$ and its values oscillate between $\kappa_{c} \approx 0$ (i.e., impermeable crack conditions) and $\kappa_{c} \approx \kappa_{m}$ (i.e., perfectly permeable crack conditions), being $\kappa_{m}$ the electric conductivity of the undamaged and the unstrained plate.

For the damaged plate $L / a=1.5$, Fig. 6 shows the influence of the crack permittivity conditions $\left(\kappa_{c} / \kappa_{m}\right)$ and the crack orientation $(\alpha)$ on the electric resistance changes. We can observe how the electric permeability of the crack is another parameter that determines the electric resistance of the plate. The more impermeable crack face conditions are, the greater is the electric resistance observed in the crack. This is easily observed in Fig. 7, where the electric potential distribution are presented for $L / a=1.5, \alpha=45^{\circ}$ and the crack permittivity conditions: (a) $\kappa_{c} / \kappa_{m}=0.0001$ and (b) $\kappa_{c} / \kappa_{m}=0.5$. 


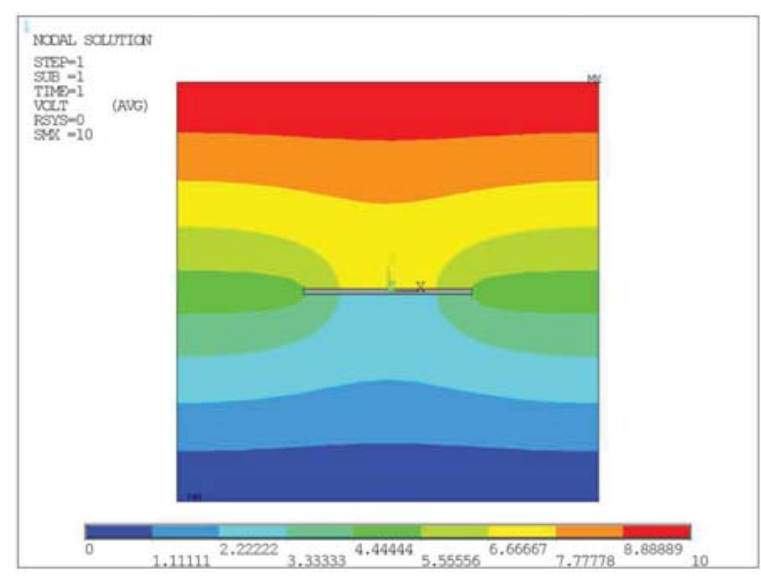

(a)

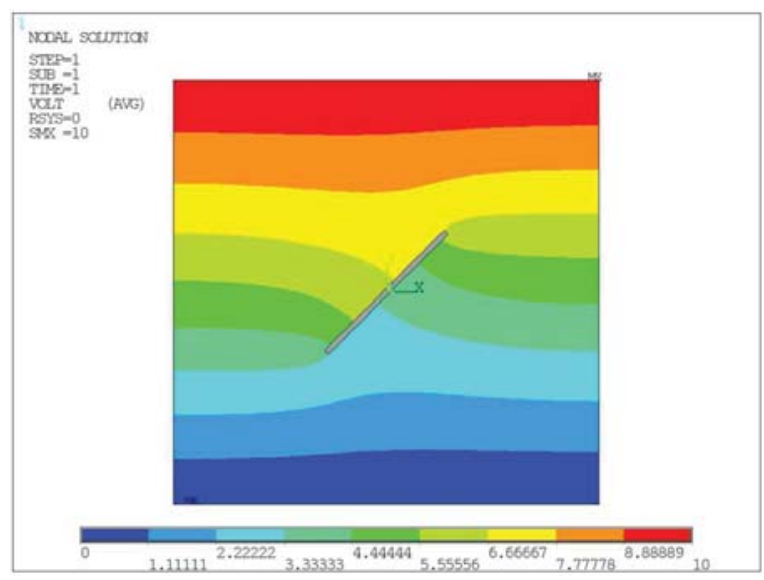

(b)

FIGURE 5. Electric potential distribution for the $L / a=2.5$ crack orientations: (a) $\alpha=0^{\circ}$ and (b) $\alpha=45^{\circ}$.

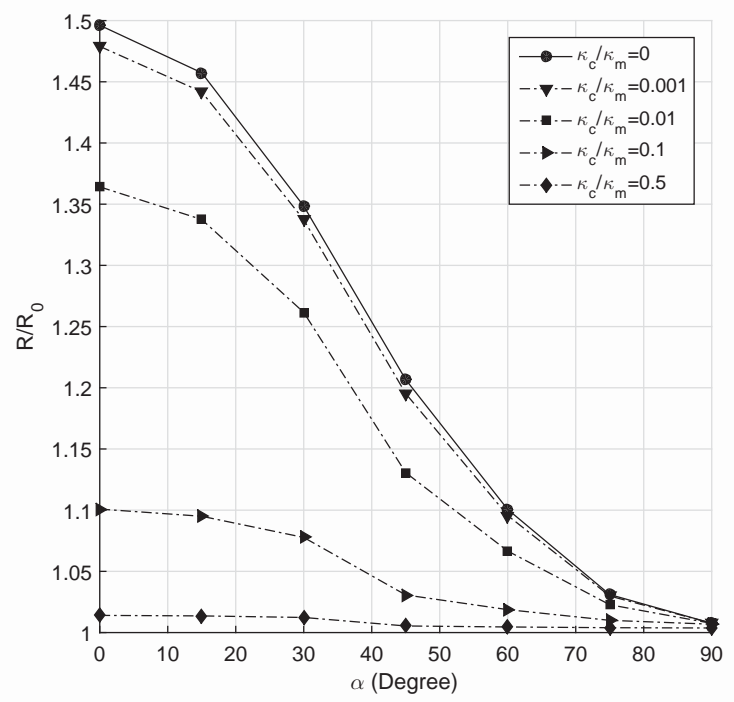

FIGURE 6. Influence of the crack permittivity conditions $\left(\kappa_{c} / \kappa_{m}\right)$ and the crack orientation $(\alpha)$ on the electric resistance changes.

\section{CONCLUSIONS}

This paper presents an eXtended Finite Element Method (XFEM)-based numerical scheme for crack detecting in composite plates computing the electrical resistivity changes caused by the presence of cracks. Using the commercial finite element package ANSYS, the virtual continuous monitoring of the structure has been solved in two steps:

- The strain response of the cracked composite plate is computed by means of the XFEM, so the electrical conductivity of the piezorresistive elements located in the domain are updated according to the strain state.

- In the second step, an increment in the electrical resistance of the plate (caused by the presence of damage) is computed by solving the electric potential field in the plate under the electrodes configuration presented in Fig.1. It turns out in an efficient virtual test to detect damage in composite panels.

In this scheme, three parameters can affect the efficiency of this virtual crack detection: the strain field around the crack alters the electric resistivity due to the piezo-resistive behavior of the MWCNT/epoxy composite, the electric permeability of the crack, the crack orientation and the severity of the damage, i.e., the crack size. 


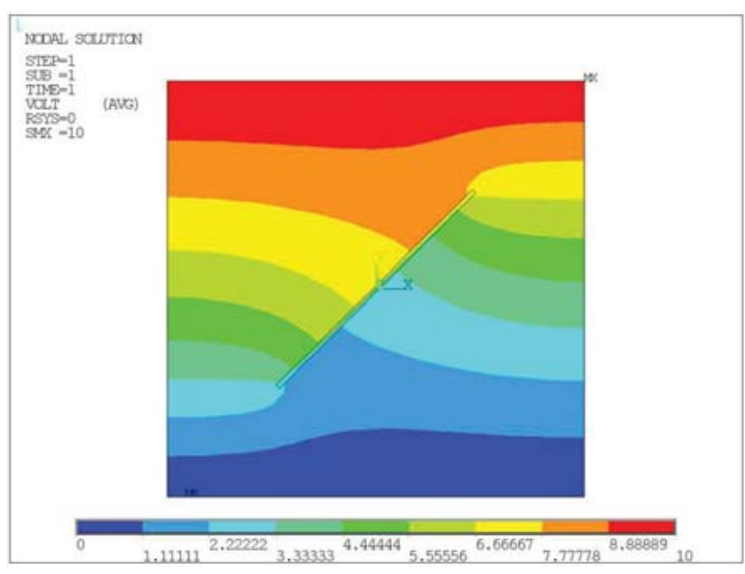

(a)

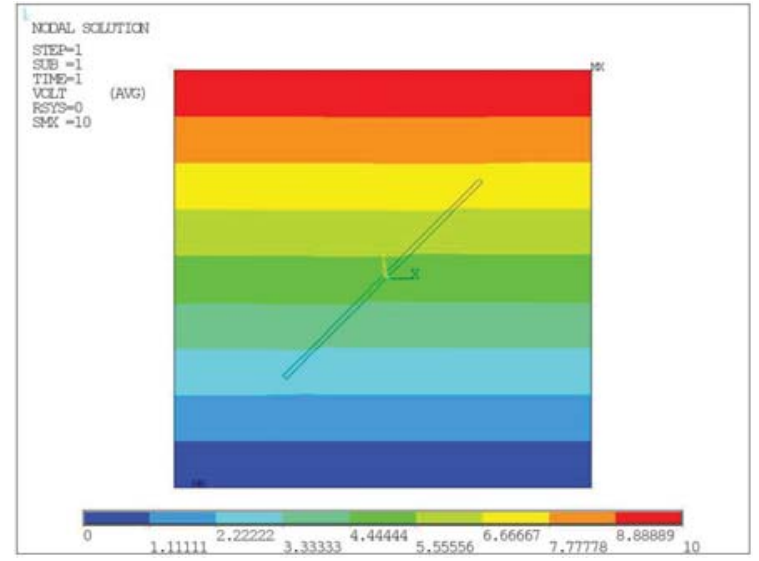

(b)

FIGURE 7. Electric potential distribution for the $L / a=1.5$ and $\alpha=45^{\circ}$ crack permittivity conditions: (a) $\kappa_{C} / \kappa_{m}=0.0001$ and (b) $\kappa_{c} / \kappa_{m}=0.5$.

Finally, the resistance changes computing scheme presented in this work could be extended for self-sensing structures developments in the civil or aeronautical industries, and it can serve as a numerical tool to assist in establishing their damage prediction protocols.

\section{ACKNOWLEDGMENTS}

This work was supported by the Ministerio de Economía y Competitividad (Spain) through the research project DPI2017-89162-R and by the Consejería de Economía, Conocimiento, Empresas y Universidades (Andalucía, Spain) through the research project P18-RT-3128. Both projects were co-funded by the European Regional Development Fund (ERDF). J. Vargas was also supported by a contract-fellowship from the Fondo Social Europeo: Empleo Joven Convocatoria 3/2019, Junta de Andalucía, Ref. EJ3-77. The financial support is gratefully acknowledged.

\section{REFERENCES}

1. E. T. Thostenson and T.-W. Chou, Nanotechnology 19, 215713 (2008).

2. X. Yu and E. Kwon, Smart Materials and Structures 18, 055010 (2009).

3. H. Zhao, Y. Zhang, P. D. Bradford, Q. Zhou, Q. Jia, F.-G. Yuan, and Y. Zhu, Nanotechnology 21, 305502 (2010).

4. Alamusi, N. Hu, H. Fukunaga, S. Atobe, Y. Liu, and J. Li, Sensors 11, 10691-10723 (2011).

5. J. Sandler, J. Kirk, I. Kinloch, M. Shaffer, and A. Windle, Polymer 44, 5893-5899 (2003).

6. A. Sanli, A. Benchirouf, C. Müller, and O. Kanoun, Sensors and Actuators, A: Physical 254, 61-68 (2017).

7. E. García-Macías, L. Rodríguez, and A. Sáez, Thin-Walled Structures 133, 27 - 41 (2018).

8. E. García-Macías, L. Rodríguez-Tembleque, A. Sáez, and F. Ubertini, Smart Materials and Structures 27, 115022 (2018).

9. L. Rodríguez-Tembleque, F. García-Sánchez, E. García-Macías, F. Buroni, and A. Sáez, Theor. Appl. Fract. Mec. 106, 102470 (2020).

10. E. García-Macías, R. Castro-Triguero, A. Sáez, and F. Ubertini, Comput. Methods Appl. Mech. Eng. 340, 396 - 423 (2018).

11. T. Belytschko and T. Black, Int. J. Numer. Methods Eng. 46, 601-620 (1999).

12. N. Sukumar, D. Chopp, N. Moes, and T. Belytschko, Comput. Methods Appl. Mech. Eng. 190, 6183-6200 (2001).

13. S. Mohammadi, Extended finite element method: For fracture analysis of structures (New York: John Wiley and Sons, 2008).

14. Fracture Analysis Guide, Release 2019 R1, ANSYS, Inc., Cannonsburg, PA (2019).

15. P. Merville, Int. J. Fract. 13, 532-534 (1977). 